\title{
Significance of Computed Tomography in the Diagnosis of Cerebrovascular Accidents
}

\author{
Sumnima Acharya ${ }^{a}$, Shyam Kishore Chaturvedi ${ }^{\mathrm{b}}$
}

\begin{abstract}
:
Introduction: Cerebrovascular Accident (CVA) is defined as abrupt onset of a neurological deficit that is attributable to a focal vascular cause. CT scan is a widely available, affordable, non-invasive and relatively accurate investigation in patients with stroke and is important to identify stroke pathology and exclude mimics. Aim of this study is to establish the diagnostic significance of computed tomography in cerebrovascular accident and to differentiate between cerebral infarction and cerebral haemorrhage with CT for better management of CVA. Methods: A one year observational cross sectional study was conducted in 100 patients that presented at the department of radiodiagnosis from emergency or ward within the one year of study period with the clinical diagnosis of stroke, and had a brain CT scan done within one to fourteen days of onset. Results: A total of 100 patients were studied. 66 were male and 34 were female with a male/female ratio of 1.9:1. Maximum number of cases (39\%) was in the age group of 61-80 yrs. Among 100 patients, 55 cases were clinically diagnosed as hemorrhagic stroke and 45 cases were clinically diagnosed with an infarct. Out of the 55 hemorrhagic cases, two cases were diagnosed as both hemorrhage and infarct by CT scan, one case had normal CT scan findings and one had subdural haemorrhage. These four cases were excluded while comparing the clinical diagnosis with CT scan finding. Among 51 clinically diagnosed cases of hemorrhagic stroke, 32(62.7\%) cases were proved by CT scan as hemorrhagic stroke and among clinically diagnosed cases of infarct, 39(86.7\%) cases were proved by CT scan as infarct which is statistically significant $(p<0.001)$. A significant agreement between clinical and CT diagnosis was observed as indicated by kappa value of 0.49 . Sensitivity, specificity, positive predictive value and negative predictive value of clinical findings as compared to CT in diagnosing hemorrhage were $84.2 \%, 67.2 \%$, $62.8 \%$ and $86.7 \%$ respectively. The accuracy of clinical diagnosis is $74 \%$. Conclusion: This study showed that CT scan is a useful diagnostic modality to identify stroke pathology and to exclude mimics.
\end{abstract}

Keywords: brain hemorrhage • brain infarction • cerebrovascular disorders • computed tomography

\section{INTRODUCTION:}

Cerebrovascular Accident (CVA) is defined by abrupt onset of a neurological deficit that is attributable to a focal vascular cause. ${ }^{1}$ The four major types of CVA are cerebral infarction, intracerebral haemorrhage $(\mathrm{ICH})$, primary subarachnoid haemorrhage (SAH) and venous occlusion. ${ }^{2}$ CVA is increasingly recognized as one of the leading cause

a - Lecturer, Department of Radiodiagnosis

Lumbini Medical College, Palpa

b - Professor and Head, Department of Radiodiagnosis

Nepalgunj Medical College, Kohalpur

Corresponding Author:

Dr. Sumnima Acharya

e-mail: doctorsumnima@gmail.com

How to cite this article:

Acharya S, Chaturvedi SK. Significance of computed tomography in the diagnosis of cerebrovascular accidents. Journal of Lumbini Medical College. 2014;2(1):18-20. doi: 10.22502/jlmc.v2i1.49. of morbidity and mortality worldwide. ${ }^{3}$ CVA is also a major health issue in semi-industrialized countries like Nepal. ${ }^{4} \mathrm{CT}$ scan is a widely available, affordable, noninvasive and relatively accurate investigation in patients with stroke and is the modality of choice as an initial investigation in such patients and is important to identify stroke pathology and exclude mimics. ${ }^{5,6}$ Therapeutic decisions regarding management of stroke require accurate diagnosis of stroke types and exclusion of mimics. Distinction between intracerebral hemorrhage (ICH) and cerebral infarction on the basis of clinical features has been shown to be unreliable. Appropriately timed CT is a safe, noninvasive gold standard, and most accurate in distinguishing ICH from cerebral infarction. $^{7}$

This type of study is the first of its kind in Western Nepal so as to provide as in depth idea about the significance of computed tomography in CVA and radiological profile of patients suffering 
from stroke in this region.

\section{METHODS:}

This is a cross-sectional study done in the department of Radiodiagnosis, Nepalgunj Medical College, Teaching Hospital, Kohalpur in one year duration from 11th July 2011 to 10th July 2012. All patients that presented at the department of radiodiagnosis from emergency or ward within one year of the study period with clinical diagnosis of stroke, and with brain CT scan done within 1 to 14 days of onset of stroke were included. History about potential risk factors was obtained like smoking, hypertension, diabetes mellitus, obesity and cardiovascular disease. Based on history and all the clinical examination findings, clinical diagnosis was made.

The patient was then subjected for noncontrast CT scan of the head performed on Siemens Somadom Emotion in supine position by taking 10 $\mathrm{mm}$ axial section with 15-20 degree angulations of the gantry to make the canthomeatal line in position. Wherever required, additional thinner cuts were taken. After this, the data was tabulated and statistically analyzed and the clinical features and various risk factors were correlated with the CT findings.

\section{RESULTS:}

A total of 100 patients were studied. Sixtysix were male and 34 were female with $\mathrm{M}: \mathrm{F}=$ 1.9. Among 100 patients, 55 cases were clinically diagnosed as hemorrhagic stroke and 45 cases were clinically diagnosed as infarct. Out of the 55 hemorrhagic cases, two cases were diagnosed as both hemorrhage and infarct, one case was normal and one had subdural haemorrhage in CT findings. All these findings are summarized in Table 1. These four cases were excluded while comparing the

Table 1: CT Scan reports in clinically suspected CVA disease

\begin{tabular}{lcc}
\hline CT Report & $\boldsymbol{n}$ & $\mathbf{\%}$ \\
\hline Hemorrhage & 36 & 36 \\
Infarct & 51 & 51 \\
Both & 2 & 2 \\
SAH & 1 & 1 \\
SDH & 1 & 1 \\
Lacunar Infarct & 6 & 6 \\
SAH and SDH & 1 & 1 \\
Large infarct and lacunar infarct & 1 & 1 \\
Normal & 1 & 1 \\
\hline Total & $\mathbf{1 0 0}$ & $\mathbf{1 0 0}$ \\
\hline
\end{tabular}

clinical diagnosis with CT scan finding. Among 51 clinically diagnosed cases of hemorrhagic stroke, 32(62.7\%) cases were proved by CT scan as hemorrhagic stroke and among clinically diagnosed cases of infarct, $39(86.7 \%)$ cases were proved by CT scan as infarct which is statistically significant ( $p<0.001)$. A significant fair agreement between clinical and CT diagnosis was observed as indicated by kappa value of 0.49 . Sensitivity, specificity, positive predictive value and negative predictive value of clinical findings as compared to $\mathrm{CT}$ in diagnosing hemorrhage were $84.2 \%, 67.2 \%, 62.8 \%$ and $86.7 \%$ respectively. The accuracy of clinical diagnosis is $74 \%$. Maximum number of cases $(39 \%)$ was in the age group of 61-80 yrs. In this study, most patients with intracerebral hemorrhage visited our hospital in subacute stage and only 5 patients with infarction visited in chronic stage (Fig.1). This study showed that maximum number of infarction $(72 \%)$ occurred in the middle cerebral artery territory, $14 \%$ were present in the anterior cerebral artery territory, $8.2 \%$ in posterior cerebral artery territory and $5.4 \%$ in basilar artery territory. In this study, out of 36 intracerebral hemorrhages, maximum number of cases $(44 \%)$ was situated in basal ganglia extending to periventricular white matter. Intraventricular extension occurred in $27.7 \%$ of cases as shown in Table 2.

\section{DISCUSSION:}

Asefa G. et al. found that in the eligible study population, the main clinical presentation was hemiparesis in $77.1 \%$ and coma in $20.8 \%$, stroke mortality was $21 \%$ and $31 \%$ had persistent neurological deficit. ${ }^{7}$ In this study, the main clinical presentation was hemiplegia in $76 \%$ and coma in $22 \%$, stroke mortality was $15 \%$ and $30 \%$ had persistent neurological deficit. These findings are comparable to those findings in other studies.

Table 2: Intracerebral hemorrhage sites of lesions in brain

\begin{tabular}{lcc}
\hline Sites of Lesions in Brain & $\boldsymbol{n}$ & $\mathbf{\%}$ \\
\hline Lentiform nucleus & 3 & 8.3 \\
$\begin{array}{l}\text { Basal ganglia extension to periventricular } \\
\text { white matter }\end{array}$ & 16 & 44 \\
External capsule involvement in CT & 2 & 5.5 \\
Frontal lobe & 7 & 19.5 \\
Temporal lobe & 8 & 22.2 \\
Parietal lobe & 10 & 27.7 \\
Occipital lobe & 0 & \\
Cerebellar hemisphere & 1 & 2.7 \\
Thalamus & 6 & 16.6 \\
\hline Ventricular extension & 10 & 27.7 \\
\hline
\end{tabular}




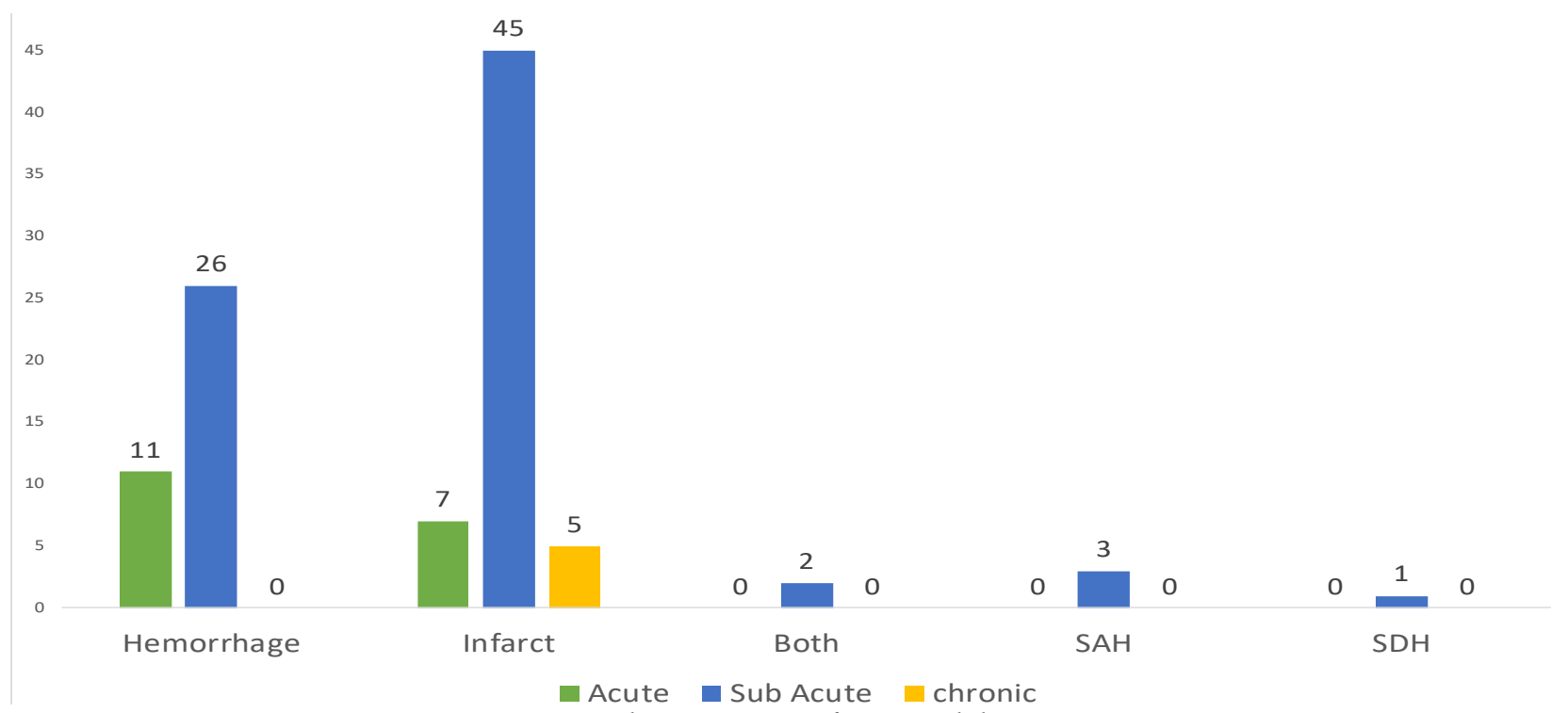

Fig 1: Duration between onset of CVA and doing CT scan.

This is the study done in western part of Nepal. In this study, maximum number of cases was of infarction that accounts for $58 \%$ of total number of cases among which six $(10 \%$ of total infarct cases) had pure lacunar infarct and one $(1.7 \%$ of total infarct) had large as well as lacunar infarct. Two cases ( $2 \%$ of total cases) had both hemorrhage and infarct. Most patients with infarction visited in sub acute stage $(45 \%)$ and only five patients with infarction visited in chronic stage. Among 58 cases, 40 were male and 18 were female with a $\mathrm{M}: \mathrm{F}=2.2$. Maximum number of cases was in the 61-80 yrs. Most of the infarct cases were in the age group of above 41 yrs. Cases below 41 yrs of age accounted for $16.6 \%$. Naik M. et al. studied 150 patients with stroke in eastern part of Nepal in BPKIHS, Dharan and found that 104 were males and 46 were females, aged 7 to 91 years in which infarction $(58 \%)$ was more common than haemorrhage $(42 \%)$ in both age group $(<40$ years and $>40$ years $) .{ }^{5}$ Shaik MM. did a study on burden of stroke in Nepal in 2012 and found that ischemic stroke is more common $(63 \%)$ than hemorrhagic stroke $(37 \%){ }^{8}$ Both studies are comparable to the findings of this study.

Sandoval J. et al. in 1999 retrospectively evaluated 200 patients of stroke. They reported hypertension as a risk factors for stroke in 13\%.9 Kaul S et al. in 2000 found that common risk factors in stroke were hypertension $(62 \%)$ and diabetes $(38 \%){ }^{10}$ Findings of the above studies are comparable to this study which were $57 \%$ and $34 \%$ respectively.

\section{CONCLUSION:}

This study showed that CT scan is an accurate imaging tool in visualizing infarcts, determining if they are bland or hemorrhagic and in visualizing intraparenchymal hemorrhage with associated edema and mass effect, as well as sub arachnoid hemorrhage.

\section{REFERENCES:}

1. Smith W, English J, Johnston S. Cerebrovascular Diseases. In: Kasper DL, Braunwald E, Fauci AS, Hauser SL, Longo DL, Jameson JL. (eds). Harrison's Principles of Internal Medicine (18 ed.). New York: Mc Graw- Hill Professional Publishing; 2008. p. 3270.

2. Osborn A. Diagnostic Neuroradiology. New Delhi(India): Mosby; 1994.

3. Salawu F, Umar I, Danburam A. Comparision of two hospital stroke scores with computed tomography in ascertaining stroke type among Nigerians. Ann Afr Med. 2009;8(1):14-8.

4. Bhalla D, Marin B, Preux PM. Stroke profile in Afghanistan and Nepal. Neurology Asia. 2009;14(2):87-94.

5. Naik M, Rauniyar RK, Sharma UK, Dwivedi S, Karki DB, Samuel J. Clinico-radiological profile of stroke in eastern Nepal: a computed tomography study. Kathmandu University Medical Journal. 2006;4(2):161-6.

6. Kolapo K, Ogun S, Danesi M, Osalusi B, Odusote K. Validation study of Siriraj stroke score in African Nigerians and evaluation of the discriminant values of its parameters. A preliminary prospective CT Scan Study. AHA Journals. 2006;37:1997-2000.

7. Asefa G, Meseret S. CT and clinical correlation of stroke diagnosis, pattern and clinical outcome among stroke patients visting Tikur Anbessa Hospital. Ethiop Med J. 2010 Apr;48(2):117-22.

8. Shaik MM, Loo KW, Gan SH. Burden of stroke in Nepal. Int J Stroke. 2012 Aug;7(6):517-20.

9. Sandoval J. Intracerebral haemorrhage in young people. Stroke. 1990;30:531-41.

10. Kaul S, Venketswamy P, Meena A, Sahay R, Murthy J. Frequency, clinical features and risk factors of lacunar infarction (data from a stroke registry in South India). Neurology India. 2000;48(2):116-19. 